\title{
DMAP-Catalyzed Reaction of Diethyl 1,3-Acetonedicarboxylate with 2-Hydroxybenzylideneindenediones: Facile Synthesis of Fluo- renone-Fused Coumarins
}

\author{
Mohanad Shkoor*(i) \\ Raghad Bayari
}

Department of Chemistry and Earth Sciences, Qatar University, P.O. Box 2713, Doha, Qatar

mshkoor@qu.edu.qa

Dedicated to Professor Adrian Schwan of the University of Guelph on the occasion of his $60^{\text {th }}$ birthday

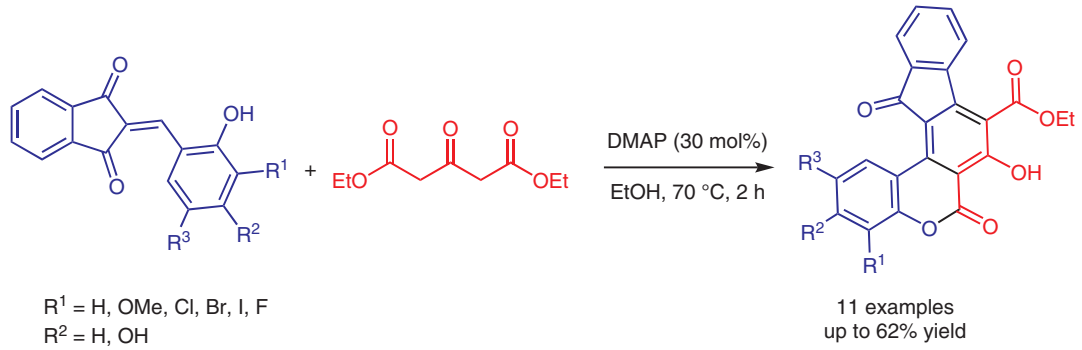

Received: 02.01.2021

Accepted after revision: 07.02.2021

Published online: 07.02 .2021

DOI: 10.1055/a-1385-2345; Art ID: st-2021-b0001-I

Abstract The base-catalyzed reaction of diethyl 1,3-acetonedicarboxylate with 2-hydroxybenzylidene indenediones was studied. The reaction provides a facile and expeditious protocol for the synthesis of natural product inspired fluorenone-fused coumarins in good to very good yields. This process resembles a combination of domino Michaelintramolecular Knoevenagel-aromatization-lactonization reactions in a single step. Although this reaction operates with many bases, the best yields were obtained with DMAP as a catalyst. This protocol could open new potential avenues for the synthesis of fused coumarins by the reaction of substituted $\beta$-keto esters with different 2-(2-hydroxybenzylidenes) of 1,3-dicarbonyl compounds.

Key words coumarin, fluorenone, indandione, DMAP, benzo[c]chromen-6-ones

Polycyclic organic motifs are abundant in synthetic and naturally occurring molecules that exhibit a diverse spectrum of applications. ${ }^{1}$ However, the complexity and diversity in their structures bring challenges to synthetic organic chemists. Therefore, the discovery and development of efficient synthetic routes to polycyclic scaffolds have been a pivotal research target. ${ }^{2-4}$

Coumarin is a privileged scaffold that is omnipresent in natural products, pharmaceuticals, and organic materials. Coumarins fused to polycyclic systems have received substantial interest from organic, ${ }^{5-9}$ medicinal, ${ }^{10-14}$ and material chemists for their unique photophysical and photochemical properties. ${ }^{15-19}$

Fluorenone (Figure 1) is an example of a polycyclic framework that is widespread in natural products. ${ }^{20}$ For instance, the natural products Gramniphenols D and E (Figure 1) exhibit anti-HIV activity. ${ }^{21}$ In addition, Caulophine (Fig- ure 1 ) displays antimyocardial ischemia activity. ${ }^{22}$ Substituted fluorenones are also abundantly present in bioactive organic molecules ${ }^{23}$ and photoelectric materials. ${ }^{24,25}$ As a result, there has been a growing interest in the synthesis of substituted fluorenones. ${ }^{26,27}$

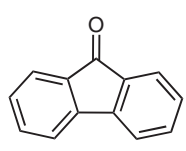

fluorenone<smiles>C=C(C)[C@H](C)COc1cc(OC)c2c(c1)C(=O)c1cc(O)cc(O)c1-2</smiles>

gramniphenols $E$<smiles>C=C(C)[C@H](C)COc1cc(OC)c2c(c1)C(=O)c1c(O)ccc(O)c1-2</smiles>

gramniphenols D

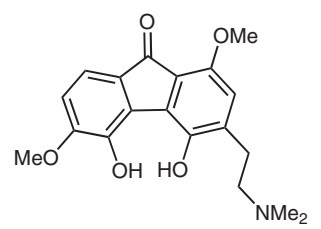

caulophine
Figure 1 Fluorenone and natural products containing fluorenone

Considering the importance of both coumarin and fluorenone, it is anticipated that molecules combining both nuclei would find applications in various fields. However, the synthesis of compounds containing fluorenone fused to coumarin has been rarely studied. Tanaka and coworkers reported the synthesis of molecules containing a fluorenone-fused coumarin core by employing transition-metal catalyst, ligand, and multistep synthesis of starting materials. $^{28}$

The base-mediated reactions of diethyl 1,3-acetonedicarboxylate with 2-hydroxychalcones have been employed in the synthesis of benzene fused coumarins (Scheme 1). ${ }^{29-31}$ 


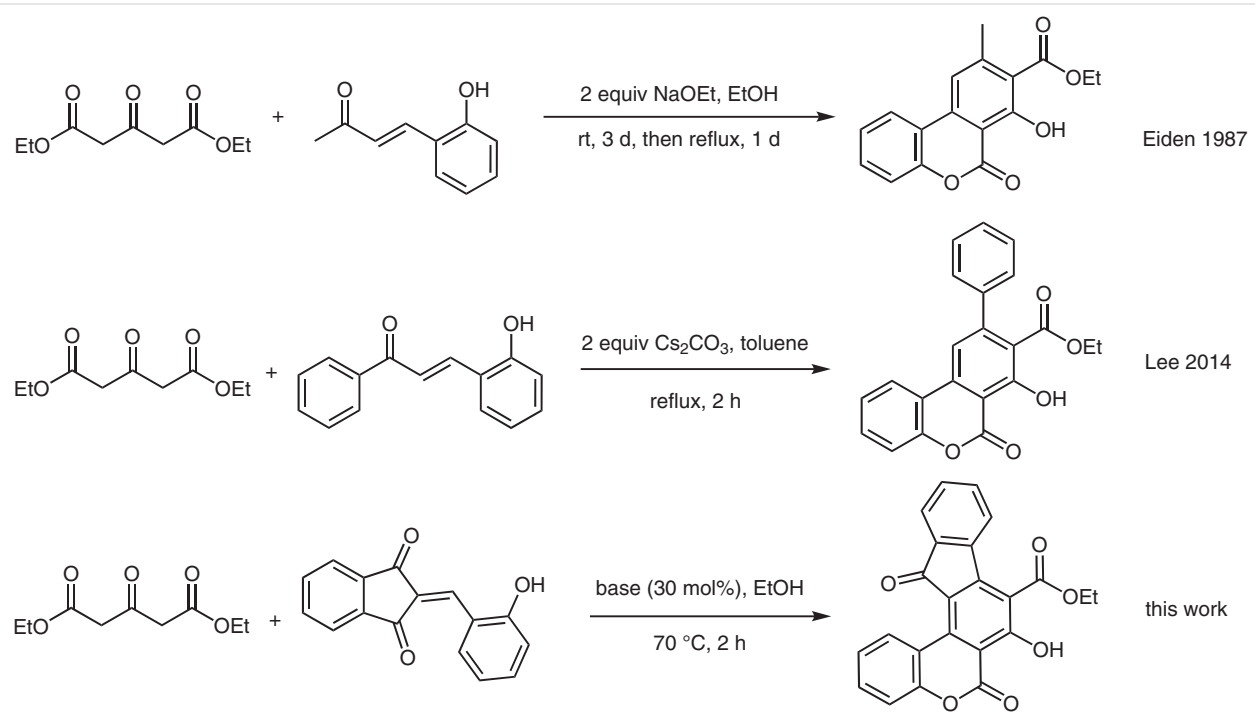

Scheme 1 Reactions of diethyl 1,3-acetonedicarboxylate with 2-hydroxychalcones

However, the reaction of substituted $\beta$-keto esters with 2-(2-hydroxybenzylidene) of both cyclic and acyclic 1,3-dicarbonyl compounds have never been studied. This type of reactions could provide a template for the synthesis of a wide variety of new fused coumarins.

In view of the importance of coumarin and fluorenone entities and in continuation of our efforts in the synthesis of fused coumarins, ${ }^{32,33}$ we report herein the base-catalyzed reaction of diethyl 1,3-acetonedicarboxylate with 2-hydroxybenzylideneindenediones leading to fluorenonefused coumarins.

Our study commenced with the preparation of the precursors 2-hydroxybenzylideneindenediones 3a-k utilizing the well-established L-proline-catalyzed condensation of 1,3-indandione (1) with substituted salicylaldehydes $\mathbf{2 a - k}$ (Scheme 2, Table 1). ${ }^{34}$<smiles>O=c1[cH+]c(=O)c2ccccc12</smiles>

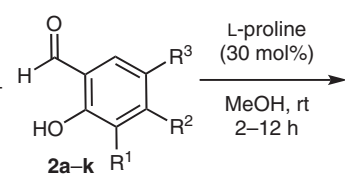<smiles>[R]c1c(P)cc(C=C2C(=O)c3ccccc3C2=O)c(O)c1Br</smiles>

Scheme 2 Synthesis of 2-hydroxybenzylideneindenediones $\mathbf{3 a - k}$

Our efforts were then directed toward the investigation of the reaction of diethyl 1,3-acetonedicarboxylate (4) with the synthesized 2-hydroxybenzylideneindenediones 3a-k. The reaction of diethyl 1,3-acetonedicarboxylate (4) with the chalcone $3 \mathbf{a}$ was chosen as a model reaction (Table 2).

In our initial experiment, 2-hydroxybenzylideneindenedione 3a was reacted with diethyl 1,3-acetonedicarboxylate in refluxing ethanol in the presence of catalytic amount (30 mol\%) of $\mathrm{Cs}_{2} \mathrm{CO}_{3}$ (entry 1, Table 2). After two hours, TLC analysis indicated completion of the reaction. Upon cooling and addition of $10 \%$ acetic acid aqueous solution, a solid precipitated. The solid was crystallized from 1,4-dioxane to afford yellowish needles in 39\% yield. The spectroscopic analysis of the product supported our proposed structure of resultant fluorenone-fused coumarin $\mathbf{5 a}$.

Motivated by this success, we then directed our efforts toward the optimization of the reaction conditions for better yields (Table 2). Thus, we tested the catalytic effect of $\mathrm{K}_{2} \mathrm{CO}_{3}$ and $\mathrm{MeCO}_{2} \mathrm{Na}$ (entries 2 and 3), both bases gave lower yields than $\mathrm{Cs}_{2} \mathrm{CO}_{3}$. The lowest yield was obtained when piperidine was used as a catalyst (entry 4), presumably due to its nucleophilicity and ability to react with hydroxybenzylideneindenediones. ${ }^{35}$ On the other hand, other organic bases such as $\mathrm{Et}_{3} \mathrm{~N}$ and L-proline improved the reaction yield (entries 5 and 6). The best yield was obtained when 4-

Table 1 Salicylaldehydes $\mathbf{2 a - k}$ and 2-Hydroxybenzylideneindenediones 3a-k Utilized in this Study

\begin{tabular}{clll}
\hline Compd & $\mathrm{R}^{1}$ & $\mathrm{R}^{2}$ & $\mathrm{R}^{3}$ \\
\hline $\mathbf{2 a}, \mathbf{3 a}$ & $\mathrm{H}$ & $\mathrm{H}$ & $\mathrm{H}$ \\
$\mathbf{2 b}, \mathbf{3 b}$ & $\mathrm{OMe}$ & $\mathrm{H}$ & $\mathrm{H}$ \\
$\mathbf{2 c}, \mathbf{3 c}$ & $\mathrm{H}$ & $\mathrm{H}$ & $\mathrm{Cl}$ \\
$\mathbf{2 d}, \mathbf{3 d}$ & $\mathrm{H}$ & $\mathrm{H}$ & $\mathrm{OMe}$ \\
$\mathbf{2 e}, \mathbf{3 e}$ & $\mathrm{H}$ & $\mathrm{H}$ & $\mathrm{Br}$ \\
$\mathbf{2 f}, \mathbf{3 f}$ & $\mathrm{H}$ & $\mathrm{H}$ & $\mathrm{I}$ \\
$\mathbf{2 g}, \mathbf{3 g}$ & $\mathrm{H}$ & $\mathrm{H}$ & $\mathrm{NO}_{2}$ \\
$\mathbf{2 h}, \mathbf{3 h}$ & $\mathrm{Br}$ & $\mathrm{H}$ & $\mathrm{Br}$ \\
$\mathbf{2 i}, \mathbf{3 i}$ & $\mathrm{Cl}$ & $\mathrm{H}$ & $\mathrm{Cl}$ \\
$\mathbf{2 j}, \mathbf{3 j}$ & $\mathrm{F}$ & $\mathrm{H}$ & $\mathrm{F}$ \\
\hline
\end{tabular}


Table 2 Screening of the Reaction Conditions for the Reaction of Diethyl 1,3-Acetonedicarboxylate $\mathbf{4}$ with Chalcone $\mathbf{3} \mathrm{a}^{\mathrm{a}}$<smiles>O=C1C(=Cc2ccccc2O)C(=O)c2ccccc21</smiles><smiles>CCOC(=O)CC(=O)CC(=O)OCC</smiles>
conditions<smiles>CCOC(=O)c1c2c(c3c(c1O)c(=O)oc1ccccc13)C(=O)c1ccccc1-2</smiles>

4

\begin{tabular}{clll}
\hline Entry & Catalyst (loading) & Solvent & Yield (\%) \\
\hline 1 & $\mathrm{Cs}_{2} \mathrm{CO}_{3}(30 \mathrm{~mol} \%)$ & $\mathrm{EtOH}$ & 39 \\
2 & $\mathrm{~K}_{2} \mathrm{CO}_{3}(30 \mathrm{~mol} \%)$ & $\mathrm{EtOH}$ & 27 \\
3 & $\mathrm{MeCO}_{2} \mathrm{Na}(30 \mathrm{~mol} \%)$ & $\mathrm{EtOH}$ & 25 \\
4 & piperidine (30 mol\%) & $\mathrm{EtOH}$ & 15 \\
5 & $\mathrm{Et}_{3} \mathrm{~N}(30 \mathrm{~mol} \%)$ & $\mathrm{EtOH}$ & 54 \\
6 & L-proline (30 mol\%) & $\mathrm{EtOH}$ & 51 \\
7 & DMAP (30 mol\%) & $\mathrm{EtOH}$ & 62 \\
8 & DMAP (10 mol\%) & EtOH & 50 \\
9 & DMAP (1 equiv) & EtOH & 40 \\
10 & - & EtOH & traces \\
11 & DMAP (30 mol\%) & EtOH & $32^{\mathrm{c}}$ \\
12 & DMAP (30 mol\%) & dioxane & 52 \\
13 & DMAP (30 mol\%) & toluene & 48 \\
14 & DMAP (30 mol\%) & MeCN & 42 \\
\hline
\end{tabular}

a Reactions were performed as: chalcone 3 a ( 1 equiv), compound $\mathbf{4}$ (1.2 equiv) at $70{ }^{\circ} \mathrm{C}$ for $2 \mathrm{~h}$.

b Isolated yield.

' Reaction was performed at room temperature. The product formed after overnight stirring.

dimethylaminopyridine (DMAP) was used to catalyze the reaction $^{36}$ (entry 7). Lowering the catalyst loading decreased the reaction yield (entry 8). When a stoichiometric amount of the catalyst was used, the reaction yield decreased (entry 9). The uncatalyzed reaction could also afford the product but with very low yield (entry 10). The desired product needed longer time to form at room temperature and the yield was relatively low (entry 11). The reaction was also successful with nonpolar and polar aprotic solvents (entries 12-14).

Several substituted 2-hydroxybenzylideneindenediones tolerated the optimized reaction conditions and gave the desired corresponding products (Table 3 ). Mono- and dihalo-substituted fluorenone-fused coumarins $\mathbf{5 c}, \mathbf{5 e}, \mathbf{5 f}, \mathbf{5 h}$, $\mathbf{5 i}$, and $\mathbf{5 j}$ were obtained in good yields. The importance of halogenation originates from a well-established drug discovery hypothesis that addition of halogens to bioactive molecules would induce antagonistic or agonist responses compared to the nonhalogenated versions of those compounds. ${ }^{37}$ Moreover, halogen substitution is a key strategy in hit-to-lead or lead optimization strategies in pharmaceutical industry as it enhances various biophysicochemical processes such as membrane binding and permeation. ${ }^{38}$ Methoxy-substituted 2-hydroxybenzylideneindenediones also tolerated the optimized reaction conditions and yielded $\mathbf{5 b}$ and $\mathbf{5 d}$ with $46 \%$ and $60 \%$ yields, respectively. In addition to that, 2-hydroxybenzylideneindenedione derived from 5-nitrosalicylaldehyde gave the corresponding nitro-substituted fluorenone-fused coumarin $\mathbf{5 g}$ in very good yield. Although the crude NMR spectrum indicated that 2,4-dihydroxybenzylideneindenedione managed to yield the desired coumarin $\mathbf{5 k}$, the compound was tedious to purify.

Table 3 Substituent Scope of the Coumarins 5a-k

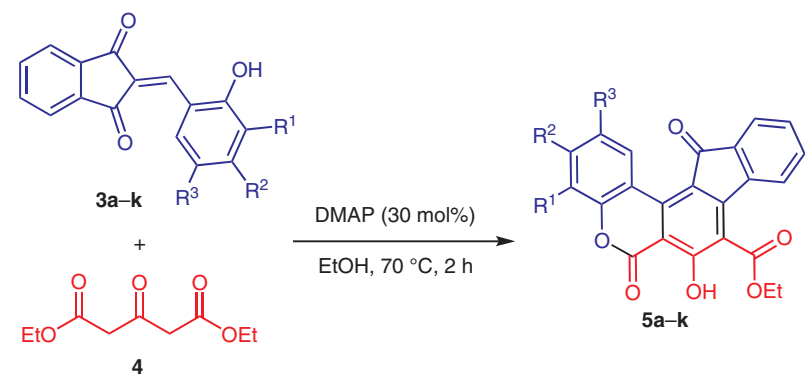

\begin{tabular}{lllll}
\hline Compd & $\mathrm{R}^{1}$ & $\mathrm{R}^{2}$ & $\mathrm{R}^{3}$ & Yield (\%) $^{\mathrm{a}}$ \\
\hline $\mathbf{5 a}$ & $\mathrm{H}$ & $\mathrm{H}$ & $\mathrm{H}$ & 62 \\
$\mathbf{5 b}$ & $\mathrm{OMe}$ & $\mathrm{H}$ & $\mathrm{H}$ & 46 \\
$\mathbf{5 c}$ & $\mathrm{H}$ & $\mathrm{H}$ & $\mathrm{Cl}$ & 55 \\
$\mathbf{5 d}$ & $\mathrm{H}$ & $\mathrm{H}$ & $\mathrm{OMe}$ & 60 \\
$\mathbf{5 e}$ & $\mathrm{H}$ & $\mathrm{H}$ & $\mathrm{Br}$ & 58 \\
$\mathbf{5 f}$ & $\mathrm{I}$ & $\mathrm{H}$ & $\mathrm{I}$ & 49 \\
$\mathbf{5 g}$ & $\mathrm{H}$ & $\mathrm{H}$ & $\mathrm{NO}_{2}$ & 58 \\
$\mathbf{5 h}$ & $\mathrm{Br}$ & $\mathrm{H}$ & $\mathrm{Br}$ & 50 \\
$\mathbf{5 i}$ & $\mathrm{Cl}$ & $\mathrm{H}$ & $\mathrm{Cl}$ & 53 \\
$\mathbf{5 j}$ & $\mathrm{F}$ & $\mathrm{H}$ & $\mathrm{F}$ & 52 \\
$\mathbf{5 k}$ & $\mathrm{H}$ & $\mathrm{OH}$ & $\mathrm{H}$ & $\sim 30$ \\
\hline
\end{tabular}

a Isolated yields.

Based on our findings and the literature, ${ }^{30}$ the reaction presumably proceeds via domino Michael-intramolecular Knoevenagel-aromatization-lactonization reactions. A tentative mechanism is depicted in Scheme 3. The Michael addition of anion $\mathbf{A}$ to the 2-hydroxybenzylideneindenedione 3a leads to the formation of the intermediate $\mathbf{B}$. Subsequent intramolecular Knoevenagel condensation and lactonization results in the formation of intermediate $\mathbf{C}$ which upon enolization and oxidation gives the desired fluorenonefused coumarin $\mathbf{5 a}$. 
<smiles>CCOC(=O)CC(=O)CC(=O)OCC</smiles><smiles>CCOC(=O)CC(=O)C(C(=O)OCC)C(c1ccccc1O)C1C(=O)c2ccccc2C1=O</smiles><smiles>CCOC(=O)CC(=O)C(CC)C(=O)OCC</smiles>

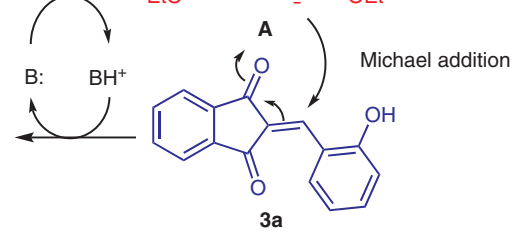

B: $\searrow-\mathrm{H}_{2} \mathrm{O}$ intramolecular Knoevenagel<smiles>CCOC(=O)C1=C2c3ccccc3C(=O)C2C2c3ccccc3OC(=O)C2C1=O</smiles>
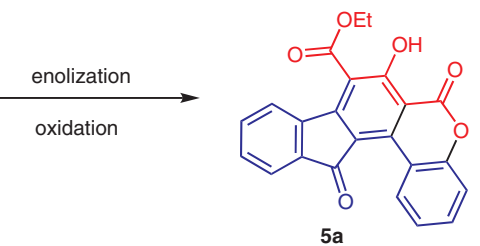

Scheme 3 A plausible mechanism for the formation of fluorenonefused coumarins $\mathbf{5 a}$

In summary, we have developed a new facile and expeditious protocol of the synthesis of substituted fluorenonefused coumarins by the base-catalyzed reaction of diethyl 1,3-acetonedicarboxylate with 2-hydroxybenzylideneindenediones. This reaction represents the first cyclization of diethyl 1,3-acetonedicarboxylate with 2-(2-hydroxybenzylidene) of 1,3-dicarbonyl compounds. The reaction operates with many bases and solvents. However, the optimized conditions required the use of DMAP as a catalyst.

\section{Funding Information}

This work was supported by Qatar University (Student Grant, Grant No. QUST-2-CAS-2019-28).

\section{Acknowledgment}

We thank Central Laboratories Unit and Environmental Science Center, Qatar University for their support in compounds analysis.

\section{Supporting Information}

Supporting information for this article is available online at https://doi.org/10.1055/a-1385-2345.

\section{References and Notes}

(1) Kancherla, S.; Jørgensen, K. B. J. Org. Chem. 2020, 85, 11140.

(2) Ibarra, I. A.; Islas-Jácome, A.; González-Zamora, E. Org. Biomol. Chem. 2018, 16, 1402.

(3) Singh, G. S.; Desta, Z. Y. Chem. Rev. 2012, 112, 6104.

(4) Kotha, S.; Meshram, M.; Tiwari, A. Chem. Soc. Rev. 2009, 38, 2065.
(5) Pratap, R.; Ram, V. J. Chem. Rev. 2014, 114, 10476.

(6) Singh, H.; Singh, J. V.; Bhagat, K.; Gulati, H. K.; Sanduja, M.; Kumar, N.; Kinarivala, N.; Sharma, S. Bioorg. Med. Chem. 2019, 27, 3477.

(7) Medina, F. G.; Marrero, J. G.; Macías-Alonso, M.; González, M. C.; Córdova-Guerrero, I.; Teissier García, A. G.; Osegueda-Robles, S. Nat. Prod. Rep. 2015, 32, 1472.

(8) Calcio Gaudino, E.; Tagliapietra, S.; Martina, K.; Palmisano, G.; Cravotto, G. RSC Adv. 2016, 6, 46394.

(9) Wang, Y.; Wang, S.; Chen, B.; Li, M.; Hu, X.; Hu, B.; Jin, L.; Sun, N.; Shen, Z. Synlett 2020, 31, 261.

(10) Al-Warhi, T.; Sabt, A.; Elkaeed, E. B.; Eldehna, W. M. Bioorg. Chem. 2020, 103, 104163.

(11) Stefanachi, A.; Leonetti, F.; Pisani, L.; Catto, M.; Carotti, A. Molecules 2018, 23, 250 .

(12) Zhang, L.; Xu, Z. Eur. J. Med. Chem. 2019, 181, 111587.

(13) Riveiro, M.; De Kimpe, N.; Moglioni, A.; Vazquez, R.; Monczor, F.; Shayo, C.; Davio, C. Curr. Med. Chem. 2010, 17, 1325.

(14) Zhu, J.-J.; Jiang, J.-G. Mol. Nutr. Food Res. 2018, 62, 1701073.

(15) Cao, D.; Liu, Z.; Verwilst, P.; Koo, S.; Jangjili, P.; Kim, J. S.; Lin, W. Chem. Rev. 2019, 119, 10403.

(16) Sun, X.; Liu, T.; Sun, J.; Wang, X. RSC Adv. 2020, 10, 10826.

(17) Tasior, M.; Kim, D.; Singha, S.; Krzeszewski, M.; Ahn, K. H.; Gryko, D. T. J. Mater. Chem. C 2015, 3, 1421.

(18) Trenor, S. R.; Shultz, A. R.; Love, B. J.; Long, T. E. Chem. Rev. 2004, 104, 3059.

(19) Miller, M. A.; Day, R. A.; Estabrook, D. A.; Sletten, E. M. Synlett 2020, 31, 450 .

(20) Shi, Y.; Gao, S. Tetrahedron 2016, 72, 1717.

(21) Hu, Q.-F.; Zhou, B.; Huang, J.-M.; Gao, X.-M.; Shu, L.-D.; Yang, G.Y.; Che, C.-T. J. Nat. Prod. 2013, 76, 292.

(22) Wang, S.; Wen, B.; Wang, N.; Liu, J.; He, L. Arch. Pharm. Res. 2009, 32, 521.

(23) Gao, H.; Wang, S.; Qi, Y.; He, G.; Qiang, B.; Wang, S.; Zhang, H. Bioorg. Med. Chem. Lett. 2019, 29, 126724.

(24) Pang, X.; Tan, Y.; Tan, C.; Li, W.; Du, N.; Lu, Y.; Jiang, Y. ACS Appl. Mater. Interfaces 2019, 11, 28246.

(25) Do, T. T.; Pham, H. D.; Manzhos, S.; Bell, J. M.; Sonar, P. ACS Appl. Mater. Interfaces 2017, 9, 16967.

(26) Revankar, H. M.; Bukhari, S. N. A.; Kumar, G. B.; Qin, H.-L.; Stefanachi, A.; Leonetti, F.; Pisani, L.; Catto, M.; Carotti, A.; Ibrar, A.; Shehzadi, S. A.; Saeed, F.; Khan, I.; Medina, F. G.; Marrero, J. G.; Macías-Alonso, M.; González, M. C.; Córdova-Guerrero, I.; Teissier, García. A. G.; Osegueda-Robles, S.; Thakur, A.; Singla, R.; Jaitak, V.; Borges, F.; Roleira, F.; Milhazes, N.; Santana, L.; Uriarte, E. Molecules 2018, 23, 250.

(27) Manick, A.-D.; Salgues, B.; Parrain, J.-L.; Zaborova, E.; Fages, F.; Amatore, M.; Commeiras, L. Org. Lett. 2020, 22, 1894.

(28) Tanaka, K.; Fukawa, N.; Suda, T.; Noguchi, K. Angew. Chem. Int. Ed. 2009, 48, 5470.

(29) Eiden, F.; Gmeiner, P. Arch. Pharm. (Weinheim, Ger.) 1987, 320, 213.

(30) Poudel, T. N.; Lee, Y. R. Org. Biomol. Chem. 2014, 12, 919.

(31) Masesane, B. I.; Mazimba, O. Bull. Chem. Soc. Ethiop. 2014, 28, 289.

(32) Shkoor, M.; Su, H.-L.; Ahmed, S.; Hegazy, S. J. Heterocycl. Chem. 2020, 57,813 .

(33) Fatunsin, O.; Iaroshenko, V.; Dudkin, S.; Shkoor, M.; Volochnyuk, D.; Gevorgyan, A.; Langer, P. Synlett 2010, 1533.

(34) Yu, J.-K.; Chien, H.-W.; Lin, Y.-J.; Karanam, P.; Chen, Y.-H.; Lin, W. Chem. Commun. 2018, 54, 9921.

(35) Pigot, C.; Noirbent, G.; Peralta, S.; Duval, S.; Nechab, M.; Gigmes, D.; Dumur, F. Helv. Chim. Acta 2019, 102, e1900229. 
(36) General Procedure for the Synthesis of Ethyl 7-Hydroxy-6,13dioxo-6,13-dihydrofluoreno[2,1-c]chromene-8-carboxylates $5 \mathbf{a}-\mathbf{k}$

4-Dimethylaminopyridine (DMAP, 0.3 equiv, $0.3 \mathrm{mmol}$ ) was added to a solution of 2-hydroxybenzylideneindenediones $\mathbf{3 a - k}$ (1 equiv, $1 \mathrm{mmol}$ ) and diethyl 1,3-acetonedicarboxylate (4, 1.2 equiv, $1.2 \mathrm{mmol})$ in ethanol $(10 \mathrm{~mL})$. The reaction solution was heated at $70{ }^{\circ} \mathrm{C}$ until completion of the reaction as indicated by TLC analysis (ca. $2 \mathrm{~h}$ ). The reaction solution was then allowed to cool down to room temperature after which an aqueous acetic acid solution (10\%) was added. The formed precipitate was filtered and the solid obtained was crystallized from dioxane.

Ethyl 7-Hydroxy-6,13-dioxo-6,13-dihydrofluoreno[2,1c]chromene-8-carboxylate (5a)

Yellow crystals; yield: $0.24 \mathrm{~g}(62 \%) ; \mathrm{mp} 239-241{ }^{\circ} \mathrm{C} .{ }^{1} \mathrm{H}$ NMR $\left(600 \mathrm{MHz}, \mathrm{CDCl}_{3}\right): \delta=1.46(\mathrm{t}, 3 \mathrm{H}, J=7.2 \mathrm{~Hz}), 4.75(\mathrm{q}, 2 \mathrm{H}, J=7.2$ $\mathrm{Hz}$ ), 7.63 (dd, $1 \mathrm{H}, J=8.2,1.2 \mathrm{~Hz}$ ), 7.40-7.44 (m, $1 \mathrm{H}), 7.45-7.55$ (m, $3 \mathrm{H}), 7.57-7.63(\mathrm{~m}, 1 \mathrm{H}), 7.75(\mathrm{dt}, 1 \mathrm{H}, J=7.3,0.9 \mathrm{~Hz}), 9.58$ (dd, $1 \mathrm{H}, J=8.3,1.5 \mathrm{~Hz}), 13.21(\mathrm{~s}, 1 \mathrm{H}) .{ }^{13} \mathrm{CNMR}(150 \mathrm{MHz}$, $\left.\mathrm{CDCl}_{3}\right): \delta=190.0,165.7,165.6,165.5,151.0,150.7,139.0,138.5$, 135.7, 134.7, 133.1, 131.6, 131.0, 125.1, 124.5, 122.9, 120.6, 117.9, 117.2, 117.0, 106.2, 62.6, 14.1. FTIR: 2988, 1698.9, 1732.7, 1666.8, 1584, 1217.7, 761.1 $\mathrm{cm}^{-1}$. Anal. Calcd for $\mathrm{C}_{23} \mathrm{H}_{14} \mathrm{O}_{6}$ : C, 71.50; $\mathrm{H}, 3.65$. Found: $\mathrm{C}, 71.58 ; \mathrm{H}, 3.68$. MS (ESI): $\mathrm{m} / z$ (\%): $386[\mathrm{M}]^{+}$(100), 341.0 (81), 312 (88), 200 (70), 341.0 (85.6).

(37) Hernandes, M.; Cavalcanti, S. M.; Moreira, D. R.; de Azevedo Junior, W. F.; Leite, A. C. Curr. Drug Targets 2010, 11, 303.

(38) Gerebtzoff, G.; Li-Blatter, X.; Fischer, H.; Frentzel, A.; Seelig, A. ChemBioChem 2004, 5, 676. 